\title{
Quality of life and acquired organ damage are intimately related to activity limitations in patients with systemic lupus erythematosus
}

\author{
Mathilda Björk ${ }^{1,2^{*}}$, Örjan Dahlström ${ }^{3}$, Jonas Wetterö ${ }^{4}$ and Christopher Sjöwall ${ }^{4}$
}

\begin{abstract}
Background: Systemic lupus erythematosus (SLE) is an autoimmune multi-organ disease, characterized by episodes of disease flares and remissions over time, which may restrain affected patients' ability to perform daily activities. The purpose of the present study was to characterize variation in activity limitations among well-defined SLE patients, and to describe disease phenotypes, acquired organ damage and their relations to activity limitation and self-reported health, respectively.
\end{abstract}

Methods: The disease phenotypes were organized into 4 different clinical groups and logistic regression analyses were used to identify how an elevated health assessment questionnaire (HAQ) score was related to disease variables such as phenotypes, disease activity and damage accrual. Correlation and multiple linear regression analyses were used to examine the association between each group of variables - background variables, disease variables and self-reported measurements - and the degree of elevated HAQ.

Results: We found a higher proportion of activity limitation in patients with skin and joint involvement compared to others. The presence of activity limitation, as detected by the HAQ instrument, was significantly associated with quality of life (EuroQol-5D) and accrual of organ damage using the Systemic Lupus International Collaborative Clinics/ACR damage index.

Conclusions: The findings highlight the differing requirements of the multi-professional rehabilitation interventions for the various SLE phenotypes in order to optimize the clinical care of the patients.

Keywords: Systemic lupus erythematosus, Disease burden, Organ damage, Disability, Quality of life, Activity limitation, Disease phenotype

\section{Background}

Systemic lupus erythematosus (SLE) is a chronic inflammatory condition characterized by multiple organ involvement, production of antibodies against nuclear constituents and deposition/formation of immune complexes in the affected organs [1]. The clinical spectrum of various disease phenotypes is remarkably diverse and this constitutes a challenge, both in terms of clinical assessment, pharmacological and non-pharmacological treatment. This is furthermore of major concern since

\footnotetext{
* Correspondence: mathilda.bjork@liu.se

${ }^{1}$ Rehabilitation Center and Department of Clinical and Experimental

Medicine, Linköping University, Linköping, Sweden

${ }^{2}$ Department of Rehabilitation, School of Health Sciences, Jönköping

University, Jönköping, Sweden

Full list of author information is available at the end of the article
}

periods of uncontrolled disease as well as medical sideeffects over time may result in irreversible organ damage [2]. The current treatment strategies with glucocorticoids and disease-modifying anti-rheumatic drugs (DMARDs) intend to relieve symptoms, induce remission, or at least allay the disease activity, prevent future flares and subsequent damage accrual [3].

SLE often affects relatively young patients who are in their most productive years of life [4], and consequences such as disability including work loss [5], activity limitations [6,7], perceived mental and physical exhaustion [8] and reduced quality of life (QoL) [9-11] are commonly found. Although new treatment options for SLE have recently become available [12], patients continue to report disability. Previous studies report that associations 
between perceived QoL and disease activity or disease severity are not clear-cut [13-16]. Instead, many authors conclude that the disease burden in SLE is multidimensional and has important physical as well as mental aspects $[11,13,17,18]$.

To be able to optimize the rehabilitation efforts for SLE patients, the knowledge about what is related to activity limitations and self-reported health needs to be expanded. Despite that more than $60 \%$ of the patients with SLE have either periodically or permanently reduced ability to perform daily activities [19], it has to our knowledge not been studied whether activity limitations and disease manifestations are related or not. An early study by Milligan et al. [20] displayed that activity limitations as measured by the health assessment questionnaire (HAQ) were not related to disease activity in female SLE patients, which could possibly be confounded by HAQ being more related to other aspects of disability [6], and/or confounders in self-reporting. A reduced health-related quality of life (HRQL) assessment in SLE patients, on the other hand, was shown to be associated with musculoskeletal impairments [21]. These findings imply that disability and self-reported health in SLE may be better explained by measures other than global disease activity, e.g., acquired organ damage, number of involved organ systems or disease phenotype.

Thus, the aims herein were to characterize variation in activity limitations among well-defined SLE patients, and to describe disease phenotypes, acquired organ damage and their relations to activity limitation and selfreported health, respectively.

\section{Methods}

\section{Patients \& laboratory analyses}

In total, 192 SLE patients included in a prospective project with structured follow-up at the Rheumatology clinic, Linköping university hospital, Sweden, were included in the present cross-sectional study. The study was based on data from the most recent visit to the rheumatologist during 2011. The patients were recruited consecutively without regard taken to present disease activity. Most were prevalent cases (93\%), but some (7 \%) had newly diagnosed SLE at the time-point of data collection. One hundred and fifty four patients $(80 \%)$ fulfilled the 1982 ACR classification criteria [22], whereas 38 (20\%) had a clinical diagnosis of SLE based on a history of abnormal antinuclear antibody (ANA) titre by immunofluorescence microscopy plus at least two typical organ manifestations (referred to as the Fries' diagnostic principle) [23]. One hundred and seventy patients (89 \%) fulfilled the 2012 Systemic Lupus International Collaborative Clinics classification criteria [24]. The study population has recently been described in detail [25].
Laboratory analyses included erythrocyte, leukocyte and platelet counts, urinalysis, erythrocyte sedimentation rate (ESR), high sensitivity C-reactive protein (CRP), creatine kinase, creatinine and plasma complement proteins $(\mathrm{C} 3$, C4). IgG class antibodies with reactivity against doublestranded (ds) DNA were detected by the Crithidia luciliae microscopy test; 56/192 (29\%) individuals were positive at the time-point of data collection, whereas altogether $44 \%$ had been anti-dsDNA antibody positive at least once (cutoff titre of 1:10, corresponding to $>99^{\text {th }}$ percentile among healthy female blood donors was used) [26].

\section{Primary outcome measure}

The validated Swedish version of HAQ [27] measuring self-reported activity limitation was the primary outcome. The HAQ consists of 20 questions representing common daily activities. The response alternatives for each of the 20 questions were 'without any difficulty' $($ score $=0)$, 'with some difficulty' (score $=1)$, 'with much difficulty' or 'with use of an assistive device' (score $=2$ ), and 'unable to do' $($ score $=3)$. The highest score obtained for any question of a given subcategory determines the score for the subcategory. A total score (0-3) was calculated based on the sum of the scores for the various subcategories divided by the number of subcategories that were answered. The Swedish version of HAQ is well-established psychometrically tested with good results [28] and widely used also in SLE [6, 29].

\section{Background variables}

Background variables concerning age, sex and disease duration were collected. The use of anti-rheumatic drugs, including antimalarials, other DMARDs and glucocorticoids was registered.

\section{Disease variables}

The number of fulfilled American Collegue of Rheumatology (ACR) criteria was registered and the study population was organized into different disease phenotypes based on the 1982 ACR classification criteria (i.e., skin disease, arthritis, renal or hematologic disorders) [22]. Acquired organ damage was estimated using the validated Systemic Lupus International Collaborative Clinics/ACR damage index (SDI), which covers 12 organ systems and measures accumulated organ damage that has occurred since the onset of SLE. SDI is scored regardless of whether the damage can be attributed to SLE or to other causes [2,30]. Disease activity was recorded by the SLE disease activity index 2000 (SLEDAI-2 K), both with and without index modification by the exclusion of laboratory items for hypocomplementemia and anti-dsDNA antibody binding [31]. In addition, the physician's global assessment (PGA; scored $0=$ remission and to $4=$ maximum disease activity) of perceived disease activity was registered [32]. 


\section{Self-reported measures}

Four patient self-reported measures were used to capture a wide range of disability and health; pain intensity, activity limitation, QoL and well-being. Pain intensity, defined as 'the experienced pain because of your SLE during the last week', was self-reported on a $0-100 \mathrm{~mm}$ visual analogue scale (VAS) ranging from 0 (no pain at all) to 100 (worst possible pain). Wellbeing was estimated in the same manner, with 0 representing 'best possible wellbeing' and 100 'worst possible wellbeing' [33]. Generic HRQL was measured using EuroQol-5D (EQ5D) that provides a profile of the self-reported problems based on five different dimensions [34]. EQ5D is useful and reliable to predict HRQL in different conditions [35].

\section{Statistical analysis}

Disease phenotypes were organized into 4 different clinical groups; skin disease (ACR criteria No. 1-3), arthritis (ACR criterion No. 5), renal disorder (ACR criterion No. 7) and hematologic disorder (ACR criterion No. 9) [22]. Differences in proportions between patients with activity limitation $(\mathrm{HAQ}>0)$ and patients without activity limitation $(\mathrm{HAQ}=0)$ were examined by Chi-square tests of proportions and differences in levels between the two groups were examined by Mann-Whitney $U$ tests.

Associations between the variables and HAQ were examined in a two-step procedure: (1) examination of variables associated with elevated HAQ score $(1=$ elevated HAQ, $0=$ no elevated HAQ), and (2) examination of variables associated with degree of elevated HAQ given that patients have elevated HAQ.

Step 1: Correlations were examined between elevated HAQ and each group of variables (background variables, disease variables and self-reported measures). To further examine the overall association between elevated HAQ and all groups of variables (background variables, disease variables and self-reported measures) the variables that correlated significantly with elevated HAQ were used in multiple logistic regression analyses, first for each group of variables and thereafter for all variables (using a stepwise forward procedure where at each step the variable which added most to the model was added until no more variable could be added with $p<0.05$ ).

Step 2: Given an elevated HAQ, correlations were then examined between degree of activity limitation and each group of variables (background variables, disease variables and self-reported measures). To further explore combined correlations (i.e., shared variance) between the variables and degree of elevated $\mathrm{HAQ}$, variables that correlated significantly with elevated HAQ were put into multiple linear regression analyses (using a stepwise forward procedure where at each step the variable which added most to the model was added until no more variable could be added with $p<0.05$ ).
All analyses were done using IBM SPSS version 20.0. $P$-values $<0.05$ were considered significant.

\section{Ethics and consent}

Oral and written informed consent was obtained from all subjects and the patient anonymity has been preserved. The study protocol was approved by the regional ethical review board in Linköping (Decision No. M7508/2008).

\section{Results}

The study population consisted of 192 patients, whereof 172 were women (mean age, 52.3 years; range, 18-87) and 20 were men (mean age, 56.0 years; range, 27-90). Data on background variables, disease variables and self-reported measures for all patients are given in Table 1. The distribution of acquired organ damage (SDI) in the study population is shown in Figure 1.

The group that reported any difficulty in performing daily activities (HAQ $>0$ ) consisted of 117 patients (61\%). This group was significantly older, used glucocorticoids to a higher extent, had more damage accrual and frequently fulfilled the ACR classification criteria for discoid rash. However, the group with elevated HAQ had less of the hematologic phenotype compared to the group where the HAQ score was zero (Table 1). In addition, the group with difficulties in performing daily activities reported significantly more pain, but lower QoL and well-being (Table 1). The distribution of patients with different phenotypes and/or elevated $v s$. no elevated HAQ scores is shown in Fig. 2; observe the high frequency of patients $(n=38)$ characterized by skin/joint involvement, without renal/hematologic phenotype, and elevated HAQ scores. Patients meeting ACR criteria for hematologic disorder had less of the arthritic phenotype ( $p=0.003$, Cramer's $\mathrm{V}=0.21$ ), and patients without difficulties in performing daily activities were more often associated with the hematologic phenotype $(p=0.002$, Cramer's V $=0.22$ ).

Using the results from distributions of cases according to phenotype and elevated HAQ scores, only the absence of hematologic phenotype was found to be significantly related to elevated HAQ in the logistic regression (Table 2). In background variables, age was significantly associated with elevated HAQ score, and among the disease variables this was also the case for SDI. In the self-reported measures, both EQ5D and well-being were significantly correlated with elevated HAQ. Finally, variables that correlated significantly with elevated $\mathrm{HAQ}$, were merged into a final logistic regression analysis (Table 2) showing a Hosmer \& Lemeshow $R^{2}$ as well as a Cox \& Snell $R^{2}$ of 0.41.

Among the patients with activity limitation, a multiple linear regression with the variables that were significantly 
Table 1 Patient characteristics. Characteristics of the patients in relation to the presence of activity limitations

\begin{tabular}{|c|c|c|c|c|}
\hline \multirow[t]{2}{*}{ Characteristics } & \multicolumn{3}{|c|}{ Mean (standard deviation) [range], or \% } & \multirow[t]{2}{*}{$p$-value } \\
\hline & Total $(n=192)$ & $\mathrm{HAQ}>0(n=117)$ & $\mathrm{HAQ}=0(n=75)$ & \\
\hline \multicolumn{5}{|l|}{ Background variables } \\
\hline Age (years) & $52.7(17.4)[18-90]$ & 56.8 (17.1) [18-90] & $46.2(16.0)[21-87]$ & $<0.0001$ \\
\hline Females & $89.6 \%$ & $90.6 \%$ & $88.0 \%$ & n.s. \\
\hline Caucasian ethnicity & $92.5 \%$ & 92.9 & 92.0 & n.s. \\
\hline Disease duration (years) & $13.4(10.2)[0-48]$ & $14.3(11.3)[0-48]$ & $12.0(8.2)[0-38]$ & n.s. \\
\hline \multicolumn{5}{|l|}{ Continuous medication } \\
\hline AM as only DMARD & $40.6 \%$ & $41.0 \%$ & $40.0 \%$ & n.s. \\
\hline Other DMARD $\pm A M$ & $29.7 \%$ & $30.8 \%$ & $28.0 \%$ & n.s. \\
\hline Glucocorticoids & $58.9 \%$ & $70.9 \%$ & $40.0 \%$ & $<0.0001$ \\
\hline \multicolumn{5}{|l|}{ Disease variables } \\
\hline Fulfilled ACR criteria ( $n$ ) & $4.6(0.3)[3-9]$ & $4.5(1.2)[3-8]$ & $4.7(1.4)[3-9]$ & n.s. \\
\hline 1. Malar rash & $43.2 \%$ & $47.0 \%$ & $37.3 \%$ & n.s. \\
\hline 2. Discoid rash & $16.1 \%$ & $20.5 \%$ & $9.3 \%$ & 0.04 \\
\hline 3. Photosensitivity & $52.6 \%$ & $54.7 \%$ & $49.3 \%$ & n.s. \\
\hline 4. Oral ulcers & $9.4 \%$ & $9.4 \%$ & $9.3 \%$ & n.s. \\
\hline 5. Arthritis & $76.6 \%$ & $76.9 \%$ & $76.0 \%$ & n.s. \\
\hline 6. Serositis & $39.1 \%$ & $36.8 \%$ & $42.7 \%$ & n.s. \\
\hline 7. Renal disorder & $20.8 \%$ & $17.1 \%$ & $26.7 \%$ & n.s. \\
\hline 8. Neurologic disorder & $4.2 \%$ & $4.3 \%$ & $4.0 \%$ & n.s. \\
\hline 9. Hematologic disorder & $51.6 \%$ & $42.7 \%$ & $65.3 \%$ & 0.002 \\
\hline 10. Immunologic disorder & $46.9 \%$ & $44.4 \%$ & $50.1 \%$ & n.s. \\
\hline 11. Antinuclear antibody* & $98.4 \%$ & $97.4 \%$ & $100 \%$ & n.s. \\
\hline SDI & $1.4(1.9)[0-8]$ & $1.9(2.1)[0-7]$ & $0.79(1.39)[0-8]$ & 0.0001 \\
\hline SLEDAI-2 K & $2.3(3.3)[0-24]$ & $2.3(3.7)[0-24]$ & $2.3(2.7)[0-11]$ & n.s. \\
\hline Modified SLEDAI** & $1.0(2.5)[0-20]$ & $1.2(2.9)[0-20]$ & $0.81(1.96)[0-10]$ & n.s. \\
\hline Physician's global assessment (PGA) & $0.26(0.55)[0-3]$ & $0.29(0.62)[0-3]$ & $0.20(0.43)[0-2]$ & n.s. \\
\hline \multicolumn{5}{|l|}{ Self-reported measures } \\
\hline Pain intensity (mm) & $29.0(27.1)[0-100]$ & 38.5 (27.0) [0-100] & $13.0(18.5)[0-73.2]$ & $<0.0001$ \\
\hline HAQ $(0-3)^{* * * *}$ & $0.44(0.60)[0-3.0]$ & $0.73(0.62)[0.13-3.0]$ & 0 & \\
\hline EQ5D & $0.67(0.30)[-0.35-1]$ & $0.56(0.31)[-0.35-1]$ & $0.84(0.18)[0-1]$ & $<0.0001$ \\
\hline Well-being (mm) & $29.8(25.8)[0-100]$ & $39.2(24.7)[0-100]$ & 13.6 (18.8) [0-74.2] & $<0.0001$ \\
\hline
\end{tabular}

*Abnormal titre of ANA by immunofluorescence microscopy

**SLEDAI- $2 \mathrm{~K}$ indicated by the exclusion of laboratory items for hypocomplementemia and anti-dsDNA antibody binding

***Performed with Mann-Whitney $U$ test or Chi-square test (where appropriate)

****Primary outcome measure

$\mathrm{AM}=$ Antimalarials; DMARD = disease modifying anti-rheumatic drugs; SDI = Systemic Lupus International Collaborative Clinics/ACR damage index; SLEDAI = SLE

disease activity index-2 K; HAQ = Health Assessment Questionnaire

$\mathrm{EQ} 5 \mathrm{D}=$ EuroQol-5D; n.s. $=$ not significant

correlated with HAQ explained $50 \%$ of the variance in HAQ scores. As demonstrated in Table 3, given an elevation of HAQ, EQ5D was identified to have the strongest relation to HAQ, followed by SDI. Even though age, pain and well-being significantly correlated with degree of elevated $\mathrm{HAQ}$, these variables could not add any significant explanation of variance in HAQ elevation than to what could be explained by EQ5D and SDI. Repeated analyses excluding patients with lowest HAQ elevation (to control for somewhat positively skewed data) yielded similar results.

\section{Discussion}

Activity limitations in the daily life constitute major problems for individuals with SLE [13]. This crosssectional cohort study of 192 well-characterized patients 


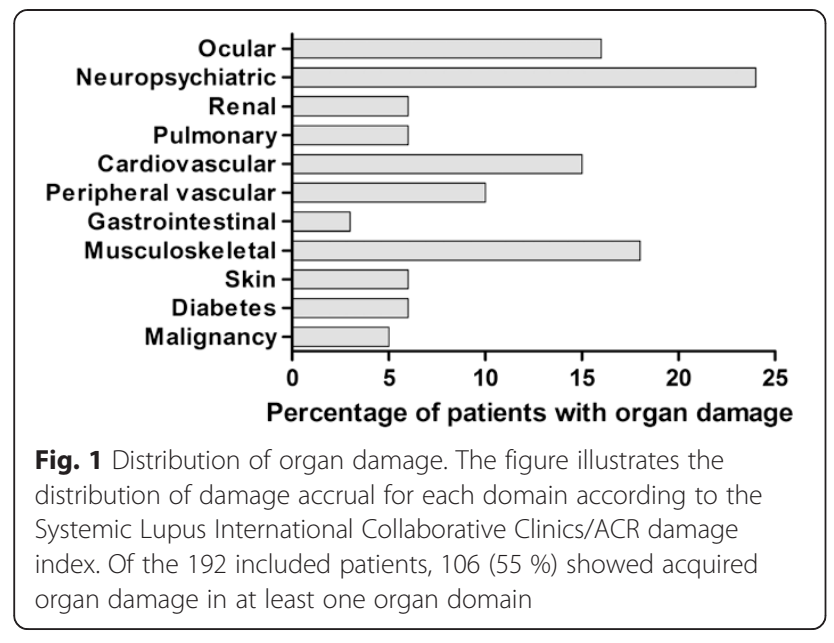

provides evidence for a higher proportion of activity limitation in SLE patients with skin and joint involvement compared to others. In addition, we found that the presence of activity limitation as detected by the HAQ instrument was significantly related to QoL (EQ5D) and acquired organ damage (SDI). Since previous research has, to our knowledge, not been focusing on the relation between organ damage and self-reported aspects in welldefined and established SLE our findings are novel. SLE patients have reported different types of discomfort related to involvement of organs and also their body to be unpredictable in how their daily activities and health are affected by the disease [36]. Our findings add knowledge to the relation between the different phenotypes of the disease and the experience of SLE in daily life. This underlines the different requirements of the multiprofessional rehabilitation interventions for the various SLE phenotypes in order to optimize the clinical care of the patients.

Although the HAQ instrument was originally developed for rheumatoid arthritis (RA) [37], it has also been employed for SLE [6, 16, 17, 20, 29], and represents a useful measure of activity limitation in disparate rheumatic conditions [27, 38-40]. In our study population the overall mean of HAQ was 0.44 , this is in line with previous results on disability in SLE $[6,29]$ and significantly altered in relation to HAQ in the Swedish general population (HAQ score of 0.08) [41]. In the study by Malcus Johnsson et al. [6], 42\% of the patients reported interference with performance of daily activities (HAQ $>0$ ) whereas we found a slightly higher proportion of patients with activity limitations (61\%). The sample in the present study was obviously older (but had similar disease duration), and this could possibly explain some of the difference. Our observation of a distinct relative increase of raised HAQ scores in individuals with skin and joint involvement is not surprising, since this lupus variant may resemble RA in many ways. Gilboe et al. used the modified HAQ instrument (MHAQ) [42] and found that activity limitations in Norwegian SLE patients were stable over time and did not predict future organ damage as was assessed by the SDI $[17,43]$.

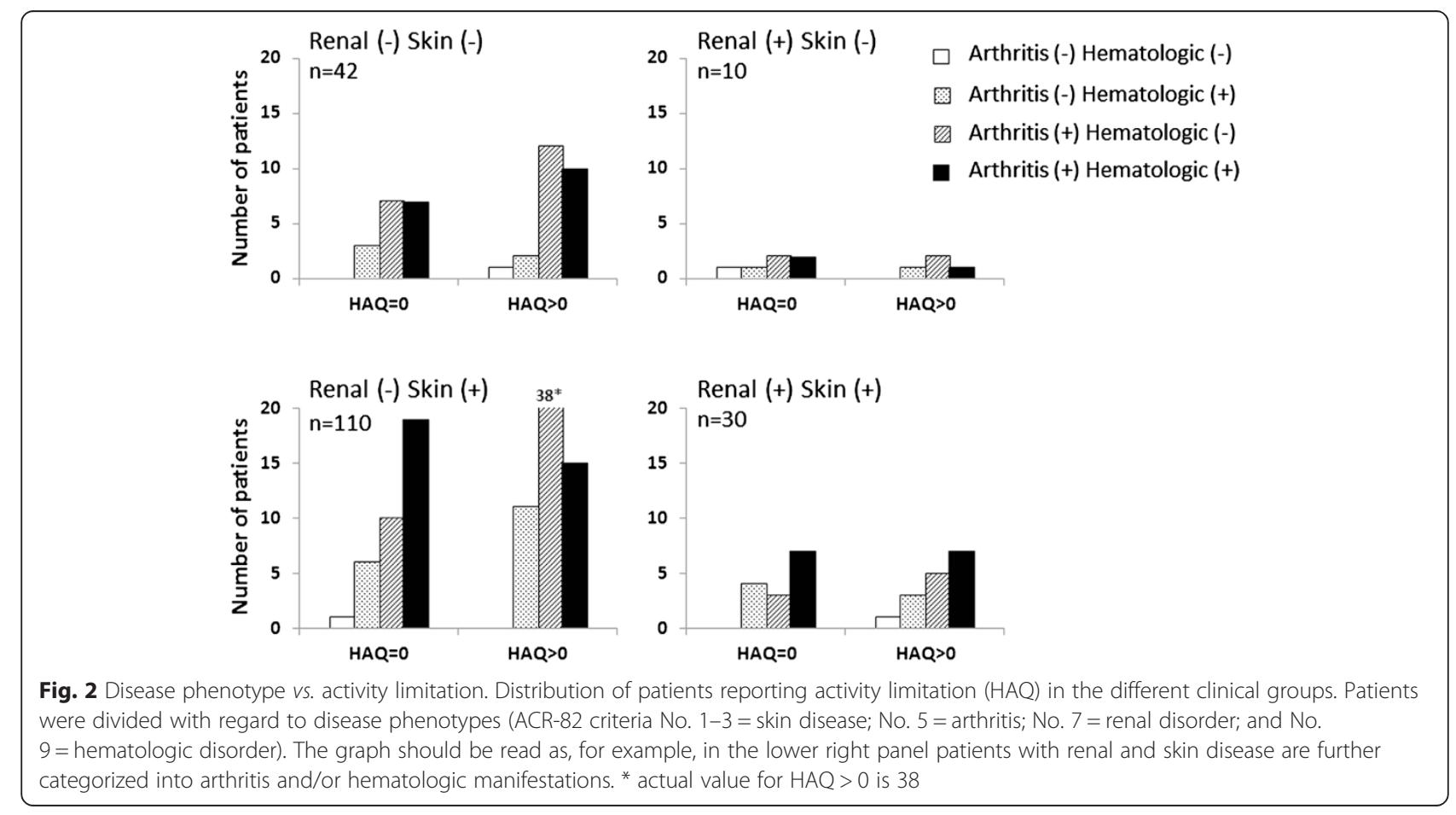


Table 2 Activity limitation predictors. Multiple logistic regression models of the effect of clinical measures on the risk of elevated HAQ score among individuals with SLE

\begin{tabular}{|c|c|c|c|c|c|c|c|c|c|}
\hline & & & $95 \%$ & Ddds Re & & $R^{2}$ & & Predic & \\
\hline & $\mathrm{B}(\mathrm{SE})$ & $P$ & $\overline{\mathrm{LL}}$ & OR & UL & $\overline{H \& L}$ & $C \& S$ & Sens. & Spec. \\
\hline Phenotypes & & & & & & 0.04 & 0.05 & 1 & 0 \\
\hline Constant & $0.947(0.231)$ & & & & & & & & \\
\hline Hematologic & $-0.926(0.306)$ & 0.002 & 0.217 & 0.396 & 0.722 & & & & \\
\hline Background variables & & & & & & 0.07 & 0.09 & 0.79 & 0.45 \\
\hline Constant & -1.49 & & & & & & & & \\
\hline Age & $0.037(0.009)$ & $<0.001$ & 1.019 & 1.038 & 1.057 & & & & \\
\hline Disease variables & & & & & & 0.06 & 0.08 & 0.66 & 0.60 \\
\hline Constant & $-0.006(0.191)$ & & & & & & & & \\
\hline SDI & $0.375(0.108)$ & $<0.001$ & 1.178 & 1.455 & 1.798 & & & & \\
\hline Self-reported measures & & & & & & 0.26 & 0.29 & 0.84 & 0.76 \\
\hline Constant & $1.891(1.318)$ & & & & & & & & \\
\hline EQ5D & $-3.276(1.502)$ & 0.029 & 0.002 & 0.038 & 0.717 & & & & \\
\hline Well-being & $0.043(0.014)$ & 0.001 & 1.017 & 1.044 & 1.072 & & & & \\
\hline Overall & & & & & & 0.41 & 0.41 & 0.87 & 0.69 \\
\hline Constant & $0.817(1.434)$ & & & & & & & & \\
\hline Age & $0.031(0.016)$ & 0.050 & 0.941 & 0.970 & 1.000 & & & & \\
\hline SDI & $0.432(0.194)$ & .0026 & 1.053 & 1.541 & 2.256 & & & & \\
\hline EQ5D & $-3.350(1.502)$ & 0.026 & 0.002 & 0.035 & 0.666 & & & & \\
\hline Well-being & $0.048(0.014)$ & $<0.001$ & 1.020 & 1.049 & 1.078 & & & & \\
\hline Hematologic & $-1.709(0.532)$ & 0.001 & 0.064 & 0.181 & 0.513 & & & & \\
\hline
\end{tabular}

$\mathrm{LL}=$ lower limit; UL = upper limit; H\&L = Hosmer \& Lemeshow; C\&S = Cox \& Snell; Sens. = sensitivity; Spec. = specificity; SDI = Systemic Lupus International Collaborative Clinics/ACR damage index; EQ5D = EuroQol-5D

Table 3 Predictors of HAQ. Factors predicting impaired high scores of HAQ among patients with elevated HAQ (Multiple Linear Regression Analysis); $n=117$ )

\begin{tabular}{llllll}
\hline & $B$ & $S E B$ & BETA & $P$ & $R$ \\
\hline Step 1 & & & & & \\
Constant & 1.444 & 0.091 & & & \\
EQ5D & -1.285 & 0.143 & -0.642 & $<0.001$ & -0.64 \\
Step 2 & & & & & \\
Constant & 1.132 & 0.106 & & & \\
SDI & 0.099 & 0.021 & 0.334 & $<0.001$ & 0.52 \\
EQ5D & -1.054 & 0.140 & -0.527 & $<0.001$ & -0.64 \\
Excluded variables & & & & & \\
Age & & & & & 0.29 \\
Well-being & & & & & 0.41 \\
Pain & & & & & 0.36
\end{tabular}

Note: $R^{2}=0.41$ for Step $1, \Delta R^{2}=0.10$ for Step $2(p<0.001)$

$B$ is the regression coefficient, SE $B$ the standard error of $B, B E T A$ is the standardized regression coefficient

$r=$ Pearson correlation between independent variables and HAQ scores
Difficulties in performing daily activities have, to our knowledge, not been reported in SLE patients with mainly skin manifestations. However, Goreshi et al. [44] reported that $62 \%$ of patients with dermatomyositis had an elevated HAQ score which was also associated with reduced QoL. Thus, based on results from these authors as well as from the present study, patients with skin disease may be underserved in terms of being evaluated by clinicians for activity limitations and self-reported health. In order to evaluate the need for rehabilitation interventions by for example by occupational therapist and physical therapists, HAQ could be used as a part of the clinical routine for patients with skin manifestations.

In the present study, organ damage (SDI), age, QoL (EQ5D) and well-being were significantly correlated with elevation of HAQ score. Among the disease phenotypes, the absence of hematologic disorder had the strongest association with a raised HAQ score. Given that the HAQ instrument is known to cover different aspects of arthritis, and that the hematologic and arthritic phenotypes were inversely associated herein, it could be speculated that it is the presence of arthritis per se, rather than the absence of hematologic disorder, which promotes this significance. 
The HAQ instrument has previously been shown to be strongly associated with well-being and QoL in SLE $[6,38]$, as well as in other rheumatic diseases [41], which is probably a sign of self-reported measures being closely related in rheumatic diseases [45]. This is seen in our results by EQ5D being highly associated with risk of elevated HAQ. In RA, however, HAQ is only weakly related to disease variables, such as the DAS28 score [41]. In line with the observation of Milligan et al., [20] we found no association between disease activity and activity limitations. In fact, SDI was the only disease variable that significantly explained variance in degree of HAQ elevation (given elevated $\mathrm{HAQ}$ ). Whereas it is rather easy to capture subtle signs of irreversible organ damage leading to activity limitations in RA by longitudinal radiographic examinations [46], the challenge is greater in SLE. SDI measures the accumulated organ damage in several organ systems that has occurred since the onset of SLE and has been present for at least 6 months regardless of its cause (i.e., caused by disease flares, therapeutic side-effects or concomitant diseases) [30]. Several studies have shown a convincing correlation between SDI and disease outcome, particularly if damage occurs early [47-49]. Thus, SDI covers a broad spectrum of symptoms and sequelae that potentially can have major impacts on the ability to perform daily activities; therefore it is not unexpected that SDI is closely associated with $\mathrm{HAQ}$. In addition, increased damage accrual was recently identified to be an important predictor of the total cost for SLE care in Sweden [50]. However, this study did not have the statistical power to consider which types of damage that were specifically associated with an elevated HAQ score. The individual SDI items have a wide range of variability, some of which are expected to affect HAQ (e.g., deforming arthritis, osteoporosis, osteonecrosis), whereas others are not expected to affect HAQ in the majority (e.g., renal, diabetes and premature gonadal failure).

Related to the finding of age as being significantly related to elevated HAQ score in SLE patients, Poole et al. [51] recently found activity limitation to be pronounced in younger parenting females with SLE. Mothers with small children $(<5$ years) reported that having energy to talk/listen to a child was the most difficult parenting task. Mothers with children older than 5 years of age reported difficulties in playing games, shopping, and doing household chores. A limitation with the HAQ instrument is that it includes only predefined activities focusing on self-care and basic needs. More recently, however, the need for incorporation of the patient perspective in assessment and interventions has been stressed [52] and assessments of a broader range of functioning, including measures of participation has been used [53, 54]. As an extension of the present study these measures could be used to for example capture the activity limitations and preferences expressed by younger women with SLE.
The large Swedish study population with well-organized data and very few internal missing values constitute the strengths of this study. However, although several important conclusions were drawn it also has limitations. In the comparison of patients with an elevated HAQ score and the patients with an unaffected $\mathrm{HAQ}$, the patients with elevation in HAQ had a higher extent of organ damage, were prescribed higher doses of glucocorticoids and were older. Age has earlier been reported to have an impact on HAQ [55], and thus it cannot be excluded that age rather than SLE per se account for some of the differences in activity limitation between the two groups. Even though HAQ is not psychometrically tested in SLE it is well established and used in earlier research and also recommended as an outcome measure in SLE [56]. The study with a cross-sectional design, within a more comprehensive prospective project, did not evaluate changes in a longitudinal perspective. To fully explore the relationship between background variables, disease variables and self-reported measures, we warrant future studies monitoring the changes in self-reported measures as disease variables changes over time. Also fatigue and depressive symptoms, as self-reported aspects often altered by SLE should be included to cover a wider range of disability.

\section{Conclusions}

In conclusion, EQ5D and the SDI were shown to have the strongest associations with activity limitations in this Swedish SLE population. These instruments record two completely different aspects of the disease, and this clearly illustrates the complexity of activity limitations in SLE.

\section{Abbreviations}

ACR: American Collegue of Rheumatology; ANA: Antinuclear antibody; CRP: C-reactive protein; DMARDs: Disease-modifying anti-rheumatic drugs; ESR: Erythrocyte sedimentation rate; EQ5D: EuroQol-5D; HAQ: Health assessment questionnaire; HRQL: Health-related quality of life; PGA: Physician's global assessment; RA: Rheumatoid arthritis; SDI: Systemic Lupus International Collaborative Clinics/ACR damage index; SLE: Systemic lupus erythematosus; SLEDAI-2K: Systemic lupus erythematosus disease activity index 2000; QoL: Quality of life; VAS: visual analogue scale.

\section{Competing interests}

The authors declare they have no competing interests.

\section{Authors' contributions}

MB was responsible of conception and design and drafted the manuscript. ÖD participated in the design of the study and performed the statistical analysis. JW has made substantial contributions to conception and design and interpretation of data. CS was responsible for data collection and made substantial contributions in the preparation of the manuscript. All authors have been involved in revising the manuscript critically and have approved the final version.

\section{Acknowledgements}

We thank research nurse Marianne Peterson and all the clinicians for their efforts. This study was financed by grants from the County Council of Östergötland, the Swedish Society for Medical Research, the Swedish Rheumatism Association, the Swedish Society of Medicine, the Professor Nanna Svartz foundation, King Gustaf V 80-year foundation, and the research foundations in memory of Clas Groschinsky and apotekare Hedberg. 


\section{Author details}

${ }^{1}$ Rehabilitation Center and Department of Clinical and Experimental Medicine, Linköping University, Linköping, Sweden. ${ }^{2}$ Department of Rehabilitation, School of Health Sciences, Jönköping University, Jönköping, Sweden. ${ }^{3}$ Swedish Institute for Disability Research, Department of Behavioural Sciences and Learning, Linköping University, Linköping, Sweden. ${ }^{4}$ Rheumatology/AIR, Department of Clinical and Experimental Medicine, Linköping University, Linköping, Sweden.

\section{Received: 29 August 2014 Accepted: 3 July 2015 \\ Published online: 12 August 2015}

\section{References}

1. Rahman A, Isenberg DA. Systemic lupus erythematosus. N Engl J Med. 2008;358:929-39.

2. Gladman DD, Urowitz MB, Rahman P, Ibanez D, Tam LS. Accrual of organ damage over time in patients with systemic lupus erythematosus. J Rheumatol. 2003;30:1955-9.

3. Dall'era M, Chakravarty EF. Treatment of mild, moderate, and severe lupus erythematosus: focus on new therapies. Curr Rheumatol Rep. 2011;13:308-16.

4. Meller S, Homey B, Ruzicka T. Socioeconomic factors in lupus erythematosus. Autoimmun Rev. 2005;4:242-6.

5. Yelin E, Tonner C, Trupin L, Gansky SA, Julian L, Katz P, et al. Longitudinal study of the impact of incident organ manifestations and increased disease activity on work loss among persons with systemic lupus erythematosus. Arthritis Care Res. 2012;64:169-75.

6. Malcus Johnsson P, Sandqvist G, Bengtsson A, Nived O. Hand function and performance of daily activities in systemic lupus erythematosus. Arthritis Rheum. 2008;59:1432-8.

7. Gallop K, Nixon A, Swinburn P, Sterling KL, Naegeli AN, Silk ME. Development of a conceptual model of health-related quality of life for systemic lupus erythematosus (SLE) from the patient's perspective. Lupus. 2012:21:934-43.

8. Bauernfeind B, Aringer M, Prodinger B, Kirchberger I, Machold K, Smolen J, et al. Identification of relevant concepts of functioning in daily life in people with systemic lupus erythematosus: A patient Delphi exercise. Arthritis Rheum. 2009:61:21-8.

9. Almehed K, Carlsten H, Forsblad-d'Elia H. Health-related quality of life in systemic lupus erythematosus and its association with disease and work disability. Scand J Rheumatol. 2010;39:58-62.

10. Sutcliffe N, Stoll T, Pyke S, Isenberg DA. Functional disability and end organ damage in patients with systemic lupus erythematosus (SLE), SLE and Sjogren's syndrome (SS), and primary SS. J Rheumatol. 1998;25:63-8.

11. Pettersson S, Lovgren M, Eriksson LE, Moberg C, Svenungsson E, Gunnarsson I, et al. An exploration of patient-reported symptoms in systemic lupus erythematosus and the relationship to health-related quality of life. Scand J Rheumatol. 2012;41:383-90.

12. Touma Z, Urowitz MB, Gladman DD. Systemic lupus erythematosus: an update on current pharmacotherapy and future directions. Expert Opin Biol Ther. 2013;13:723-37.

13. Kiani AN, Petri M. Quality-of-life measurements versus disease activity in systemic lupus erythematosus. Curr Rheumatol Rep. 2010;12:250-8.

14. Freire EA, Maia IO, Nepomuceno JC, Ciconelli RM. Damage index assessment and quality of life in systemic lupus erythematosus patients (with long-term disease) in Northeastern Brazil. Clin Rheumatol. 2007;26:423-8.

15. Wang C, Mayo NE, Fortin PR. The relationship between health related quality of life and disease activity and damage in systemic lupus erythematosus. J Rheumatol. 2001;28:525-32.

16. Benitha R, Tikly M. Functional disability and health-related quality of life in South Africans with rheumatoid arthritis and systemic lupus erythematosus. Clin Rheumatol. 2007:26:24-9.

17. Gilboe IM, Kvien TK, Husby G. Health status in systemic lupus erythematosus compared to rheumatoid arthritis and healthy controls. J Rheumatol. 1999;26:1694-700.

18. Nuttall A, Isenberg DA. Assessment of disease activity, damage and quality of life in systemic lupus erythematosus: new aspects. Best Pract Res Clin Rheumatol. 2013;27:309-18.

19. Boomsma MM, Bijl M, Stegeman CA, Kallenberg CG, Hoffman GS, Tervaert JW. Patients' perceptions of the effects of systemic lupus erythematosus on health, function, income, and interpersonal relationships: a comparison with Wegener's granulomatosis. Arthritis Rheum. 2002;47:196-201.
20. Milligan SE, Hom DL, Ballou SP, Persse LJ, Svilar GM, Coulton CJ. An assessment of the Health Assessment Questionnaire functional ability index among women with systemic lupus erythematosus. J Rheumatol. 1993;20:972-6.

21. Zhu TY, Tam LS, Lee WW, Lee KK, Li EK. Relationship between flare and health-related quality of life in patients with systemic lupus erythematosus. J Rheumatol. 2010;37:568-73.

22. Tan EM, Cohen AS, Fries JF, Masi AT, McShane DJ, Rothfield NF, et al. The 1982 revised criteria for the classification of systemic lupus erythematosus. Arthritis Rheum. 1982;25:1271-7.

23. Fries JF, Holman HR. Systemic lupus erythematosus: a clinical analysis. Major Probl Intern Med. 1975;6:v-199.

24. Petri M, Orbai AM, Alarcon GS, Gordon C, Merrill JT, Fortin PR, et al. Derivation and validation of the Systemic Lupus International Collaborating Clinics classification criteria for systemic lupus erythematosus. Arthritis Rheum. 2012;64:2677-86.

25. Ighe A, Dahlstrom O, Skogh T, Sjowall C. Application of the 2012 Systemic Lupus International Collaborating Clinics classification criteria to patients in a regional Swedish systemic lupus erythematosus register. Arthritis Res Ther. 2015;17:3.

26. Enocsson H, Sjowall C, Wirestam L, Dahle C, Kastbom A, Ronnelid J, et al. Four Anti-dsDNA Antibody Assays in Relation to Systemic Lupus Erythematosus Disease Specificity and Activity. J Rheumatol. 2015;42:817-25.

27. Ekdahl C, Eberhardt K, Andersson SI, Svensson B. Assessing disability in patients with rheumatoid arthritis. Use of a Swedish version of the Stanford Health Assessment Questionnaire. Scand J Rheumatol. 1988;17:263-71.

28. Lomi C, Burckhardt C, Nordholm L, Bjelle A, Ekdahl C. Evaluation of a Swedish version of the arthritis self-efficacy scale in people with fibromyalgia. Scand J Rheumatol. 1995:24:282-7.

29. Colangelo KJ, Pope JE, Peschken C. The minimally important difference for patient reported outcomes in systemic lupus erythematosus including the HAQ-DI, pain, fatigue, and SF-36. J Rheumatol. 2009;36:2231-7.

30. Gladman D, Ginzler E, Goldsmith C, Fortin P, Liang M, Urowitz M, et al. The development and initial validation of the Systemic Lupus International Collaborating Clinics/American College of Rheumatology damage index for systemic lupus erythematosus. Arthritis Rheum. 1996;39:363-9.

31. Gladman DD, Ibanez D, Urowitz MB. Systemic lupus erythematosus disease activity index 2000. J Rheumatol. 2002;29:288-91.

32. Scott DL. A simple index to assess disease activity in rheumatoid arthritis J Rheumatol. 1993;20:582-4.

33. Hallert E, Thyberg I, Hass U, Skargren E, Skogh T. Comparison between women and men with recent onset rheumatoid arthritis of disease activity and functional ability over two years (the TIRA project). Ann Rheum Dis. 2003:62:667-70.

34. Leidl R. Preferences, quality of life and public health. Eur J Public Health. 2009;19:228-9.

35. Dolan P. Modeling valuations for EuroQol health states. Med Care. 1997:35:1095-108.

36. Mattsson M, Moller B, Stamm T, Gard G, Bostrom C. Uncertainty and opportunities in patients with established systemic lupus erythematosus: a qualitative study. Musculoskeletal Care. 2012;10:1-12.

37. Fries JF, Spitz P, Kraines RG, Holman HR. Measurement of patient outcome in arthritis. Arthritis Rheum. 1980;23:137-45.

38. Hersh A. Measures of health-related quality of life in pediatric systemic lupus erythematosus: Childhood Health Assessment Questionnaire (C-HAQ), Child Health Questionnaire (CHQ), Pediatric Quality of Life Inventory Generic Core Module (PedsQL-GC), Pediatric Quality of Life Inventory Rheumatology Module (PedsQL-RM), and Simple Measure of Impact of Lupus Erythematosus in Youngsters (SMILEY). Arthritis Care Res. 2011;63 Suppl 11:S446-53.

39. Husted JA, Gladman DD, Farewell VT, Cook RJ. Health-related quality of life of patients with psoriatic arthritis: a comparison with patients with rheumatoid arthritis. Arthritis Rheum. 2001;45:151-8.

40. Tollisen A, Sanner H, Flato B, Wahl AK. Quality of life in adults with juvenile-onset dermatomyositis: a case-control study. Arthritis Care Res. 2012;64:1020-7.

41. Bjork MA, Thyberg IS, Skogh T, Gerdle BU. Hand function and activity limitation according to health assessment questionnaire in patients with rheumatoid arthritis and healthy referents: 5-year followup of predictors of activity limitation (The Swedish TIRA Project). J Rheumatol. 2007;34:296-302.

42. Pincus T, Summey JA, Soraci Jr SA, Wallston KA, Hummon NP. Assessment of patient satisfaction in activities of daily living using a modified Stanford Health Assessment Questionnaire. Arthritis Rheum. 1983;26:1346-53. 
43. Gilboe IM, Kvien TK, Husby G. Disease course in systemic lupus erythematosus: changes in health status, disease activity, and organ damage after 2 years. J Rheumatol. 2001;28:266-74.

44. Goreshi R, Chock M, Foering K, Feng R, Okawa J, Rose M, et al. Quality of life in dermatomyositis. J Am Acad Dermatol. 2011;65:1107-16.

45. Thyberg I, Skogh T, Hass UA, Gerdle B. Recent-onset rheumatoid arthritis: a 1-year observational study of correlations between health-related quality of life and clinical/laboratory data. J Rehabil Med. 2005;37:159-65.

46. Clarke AE, St-Pierre Y, Joseph L, Penrod J, Sibley JT, Haga M, et al. Radiographic damage in rheumatoid arthritis correlates with functional disability but not direct medical costs. J Rheumatol. 2001;28:2416-24.

47. Rahman P, Gladman DD, Urowitz MB, Hallett D, Tam LS. Early damage as measured by the SLICC/ACR damage index is a predictor of mortality in systemic lupus erythematosus. Lupus. 2001;10:93-6.

48. Nived O, Jonsen A, Bengtsson AA, Bengtsson C, Sturfelt G. High predictive value of the Systemic Lupus International Collaborating Clinics/American College of Rheumatology damage index for survival in systemic lupus erythematosus. J Rheumatol. 2002;29:1398-400.

49. Stoll T, Seifert B, Isenberg DA. SLICC/ACR Damage Index is valid, and renal and pulmonary organ scores are predictors of severe outcome in patients with systemic lupus erythematosus. Br J Rheumatol. 1996;35:248-54.

50. Jonsen A, Bengtsson AA, Hjalte F, Petersson IF, Willim M, Nived O: Total cost and cost predictors in systemic lupus erythematosus - 8-years follow-up of a Swedish inception cohort. Lupus 2015. May 8. In press, doi: 10.1177/ 0961203315584812.

51. Poole JL, Rymek-Gmytrasiewicz M, Mendelson C, Sanders M, Skipper B. Parenting: the forgotten role of women living with systemic lupus erythematosus. Clin Rheumatol. 2012:31:995-1000.

52. Schmeding A, Schneider M. Fatigue, health-related quality of life and other patient-reported outcomes in systemic lupus erythematosus. Best Pract Res Clin Rheumatol. 2013:27:363-75.

53. Katz P, Morris A, Trupin L, Yazdany J, Yelin E. Disability in valued life activities among individuals with systemic lupus erythematosus. Arthritis Rheum. 2008:59:465-73.

54. Andrews JS, Trupin L, Schmajuk G, Barton J, Margaretten M, Yazdany J, et al. Muscle Strength, Muscle Mass, and Physical Disability in Women with Systemic Lupus Erythematosus. Arthritis Care Res. 2014;67:120-7.

55. Krishnan E, Sokka T, Hakkinen A, Hubert H, Hannonen P. Normative values for the Health Assessment Questionnaire disability index: benchmarking disability in the general population. Arthritis Rheum. 2004;50:953-60.

56. Feld J, Isenberg D. Why and how should we measure disease activity and damage in lupus? Presse Med. 2014;43:e151-6.

\section{Submit your next manuscript to BioMed Central and take full advantage of:}

- Convenient online submission

- Thorough peer review

- No space constraints or color figure charges

- Immediate publication on acceptance

- Inclusion in PubMed, CAS, Scopus and Google Scholar

- Research which is freely available for redistribution 